VOL. 43 (1991) [257-264]

\title{
AUTOMORPHISMS OF DIRECTED TRIPLE SYSTEMS
}

\author{
Charles J. Colbourn
}

The obvious necessary conditions for a triple system of index two having a given automorphism group to underly a directed triple system having the same group are shown to be sufficient. An efficient algorithm for directing the triple system in a way that preserves its group is given. Applications to the existence of directed triple systems with various automorphisms are outlined.

\section{BACKGROUND}

A triple system of order $v$ and index $\lambda$, denoted $T S(v, \lambda)$, is a $v$-set $V$ of elements, together with a collection $\mathcal{B}$ of 3-element subsets of $V$, called triples; moreover, every 2-subset of $V$ appears in precisely $\lambda$ triples. A partial $T S(v, \lambda)$, or $\operatorname{PTS}(v, \lambda)$, relaxes the requirement so that every 2 -subset must appear in at most $\lambda$ triples. Triples systems have been widely studied for over a century; see [10] for numerous references.

Hung and Medelsohn [13] considered triple systems in which the triples are ordered. A transitive triple $(x, y, z)$ is taken to contain the ordered pairs $\{(x, y),(x, z),(y, z)\}$. A directed triple system of order $v$ and index $\lambda$, or $\operatorname{DTS}(v, \lambda)$, is a $v$-set $V$ of elements, and a collection $\mathcal{B}$ of transitive triples, having the property that every ordered pair $(x, y)$ of distinct elements appears in precisely $\lambda$ of the transitive triples in $\mathcal{B}$. A partial $\operatorname{DTS}(v, \lambda)$ has every ordered pair in at most $\lambda$ of the transitive triples.

Necessary and sufficient conditions for the existence of directed triple systems have been established by Hung and Mendelsohn for $\lambda=1$ [13], and by Seberry and Skillicorn for all $\lambda[14]$; they found that the conditions for the existence of a $D T S(v, \lambda)$ are the same as those for the existence of a $T S(v, 2 \lambda)$. However, a much stronger relationship holds, as we shall see.

Naturally, if one treats the transitive triples of a $\operatorname{DTS}(v, \lambda)$ as unordered 3-subsets, one obtains a $T S(v, 2 \lambda)$; this is the underlying triple system of the DTS. The underlying triple system of a $D T S$ is termed directable, and the operation of producing the $D T S$ from the $T S$ is directing.

\section{Received 20 April 1990}

Thanks to Alex Rosa for assistance in locating references. Research of the author is supported by NSERC Canada under grant number A0579.

Copyright Clearance Centre, Inc. Serial-fee code: 0004-9729/91 \$A2.00+0.00. 
Theorem A. $[4,5,12]:$ Every $T S(v, 2 \lambda)$ is directable.

The existence theorems for DTS are simple corollaries of Theorem A, together with existence results for $T S(v, \lambda)$ 's (see, for example, [15]). Moreover, many natural problems on directed triple systems can be solved by employing the solution for the analogous problem on triple systems with even index, and directing the triple systems using Theorem A. Any property preserved in directing a triple system can be studied in this way; see $[4,5]$ for some examples.

The automorphism group of a DTS necessarily appears as a subgroup of the automorphism group of its underlying triple system. However, the automorphisms of the triple system need not be preserved by the directing operation; there is, for example, a $\operatorname{DTS}(7,1)$ having only the trivial automorphism but whose underlying $T S(7,2)$ has 168 automorphisms [6].

We consider the following question. Suppose that there exists a $T S(v, 2 \lambda)$ having $\Gamma$ as an automorphism group, where $\Gamma$ need not be the full automorphism group of the $T S$. When can the $T S(v, 2 \lambda)$ be directed as a $D T S(v, \lambda)$ having $\Gamma$ as an automorphism group?

The motivation for the question is perhaps obvious, but let us mention a few points here. There has been much work on cyclic designs whose automorphism group contains a $v$-cycle [9], and on $k$-rotational designs, whose automorphism group contains a permutation fixing one element and mapping the remaining elements in $k$ cycles of length $(v-1) / k$. Existence for cyclic $\operatorname{DTS}(v, 1)$ has been settled by Colbourn and Colbourn [8], and existence of cyclic $\operatorname{DTS}(v, \lambda)$ for all $\lambda$ by Cho, Han and Kang [3]. Cho, Chae and Hwang [2] settled existence for $k$-rotational $D T S(v, 1)$ 's. In all of these cases, the constructions are remarkably similar to analogous constructions for triple systems, and hence one might expect that the existence of DTS with certain automorphism groups is a simple consequence of the existence of underlying triple systems with the same group. However, we shall see that a $T S(v, 2 \lambda)$ can have automorphisms that cannot be shared by any $D T S(v, \lambda)$ based on it. Nevertheless, a first indication that some general correspondence exists is given by Harms [12]. She determines when it is possible to direct a cyclic $T S(v, 2)$ to obtain a cyclic $D T S(v, 1)$. Our objective is to establish a general correspondence that permits results about the structure of the automorphisms of triple systems to be carried over to directed triple systems, and in this way to provide simplified proofs of earlier results for automorphisms of directed triple systems, and to carry over known results from triple systems to directed triple systems.

\section{FUll ORBIT PRESENTATIONS}

Let $(V, \mathcal{B})$ be a partial triple system $\operatorname{PTS}(v, 2 \lambda)$, and let $\Gamma$ be an automorphism group of the PTS. For a triple $t=\{x, y, z\}$ in $\mathcal{B}$, let $\Gamma_{t}$ be the set of automorphisms 
of $\Gamma$ restricted to the elements $\{x, y, z\} . \Gamma_{t}$ is a subgroup of $S_{3}$; hence $\gamma_{t}=\left|\Gamma_{t}\right|$ is 1 , 2,3 or 6 . The triple $t$ is said to be of type $\gamma_{t}$.

We first prove a simple necessary condition:

Lemma 2.1. If a $T S(v, 2 \lambda)(V, \mathcal{B})$ with group $\Gamma$ is directable as a $\operatorname{DTS}(v, \lambda)$ with group $\Gamma$, then for each triple $t$ of the TS $(v, 2 \lambda)$, the number of occurrences of the triple $t$ is a multiple of $\gamma_{t}$.

Proof: It is easily verified that for any transitive triple $t=(x, y, z)$, the action of $\Gamma_{t}$ on $t$ yields $\gamma_{t}$ distinct transitive triples (but of course the same underlying unordered triple).

This lemma generalises the observation that a cyclic $T S(v, 2)$ cannot underly a cyclic $\operatorname{DTS}(v, 1)$ when $v \equiv 0(\bmod 3)$, since in that case the (required) block $\{0, v / 3,2 v / 3\}=t$ has $\gamma_{t} \equiv 0(\bmod 3)$.

Another way to develop the necessary condition given in Lemma 2.1 is perhaps more instructive. In the usual way, we define the orbit of a triple $t=\{x, y, z\}$ under $\Gamma, \operatorname{Orb}(t, \Gamma)$, to be the set of all triples

$$
\{\{\pi x, \pi y, \pi z\}: \pi \in \Gamma\} .
$$

The orbit of a transitive triple $t^{\prime}=(x, y, z)$,

$$
\operatorname{Orb}\left(t^{\prime}, \Gamma\right)=\{(\pi x, \pi y, \pi z) ; \pi \in \Gamma\}
$$

For a triple $t=\{x, y, z\}$, we also define the full orbit of $t$ under $\Gamma, F O r b(t, \Gamma)$, to be the multiset

$$
\{\{a, b, c\}:(a, b, c) \in \operatorname{Orb}((x, y, z), \Gamma)\} .
$$

For example, if $\Gamma=\langle(x y z)\rangle, \operatorname{Orb}(\{x, y, z\}, \Gamma)=\{\{x, y, z\}\} ;$ however, $\operatorname{Orb}((x, y, z), \Gamma)=\{(x, y, z),(y, z, x),(z, y, x)\}$ and hence $\operatorname{FOrb}(\{x, y, z\}, \Gamma)$ $=\{\{x, y, z\}\{x, y, z\},\{x, y, z\}\}$. In general, $F \operatorname{Forb}(t, \Gamma)$ consists of $\gamma_{t}$ copies of $\operatorname{Orb}(t, \Gamma)$.

Let $(V, \mathcal{B})$ be a $T S(v, 2 \lambda)$ having $\Gamma$ as an automorphism group. For this $T S$ to be directable as a $D T S(v, \lambda)$ having $\Gamma$ as an automorphism group, the Lemma 2.1 requires that there be a multiset of triples $\left\{t_{1}, \cdots, t_{s}\right\} \subseteq \mathcal{B}$, so that the multiset union of $\operatorname{FOrb}\left(t_{i}, \Gamma\right)$ for $i=1, \cdots$, s gives the multiset of triples $\mathcal{B}$. We call such a collection $\left\{t_{1}, \cdots, t_{\text {, }}\right\}$ a full orbit presentation of $(V, \mathcal{B})$ under $\Gamma$. Lemma 2.1 is an easy consequence of the fact that every $\operatorname{DTS}(v, \lambda)$ with group $\Gamma$ has an underlying $T S(v, 2 \lambda)$ with a full orbit presentation under $\Gamma$.

A further necessary condition can be derived. Suppose that $\Gamma$ contains an automorphism $\pi$ fixing two points, $x$ and $y$, of a triple, but moves the third point $z$ of the 
triple. For $\pi$ to be preserved, all triples in the orbit must contain the pair $\{x, y\}$ in the same direction. Now if $\pi$ has order $2 \lambda$ (or any order greater than $\lambda$ ), the automorphism $\pi$ cannot be preserved in the DTS, since the pair $(x, y)$ or the pair $(y, x)$ must then appear more than $\lambda$ times.

For $\lambda=2$, this requirement simplifies: there is no $\pi \in \Gamma$ that fixes two points of a triple and moves the third; when this condition is met, we say that $\Gamma$ has a planar action on the TS.

\section{Directing a full orbit presentation}

We now show that the presence of a full orbit presentation is not only necessary for directability, it is also sufficient in the case that $r$ has a planar action.

THEOREM 3.1. Let $\left\{t_{1}, \cdots, t_{s}\right\}$ be a full orbit presentation under automorphism group $\Gamma$ of a $T S(v, 2 \lambda)(V, \mathcal{B})$. Suppose that $\Gamma$ has a planar action on $(V, \mathcal{B})$. Then $(V, \mathcal{B})$ is the underlying triple system of a $\operatorname{DTS}(v, \lambda)$ with automorphism group $\Gamma$.

PROOF: Let $p_{1}, \cdots, p_{r}$ be representatives for the orbits under $\Gamma$ of unordered pairs of elements of $V$. Using the orbits of pairs and the full orbits of triples, we form a graph $G$ as follows.

1. For each pair $p_{i}=\left\{a_{i}, b_{i}\right\}$ (representing an orbit under $\Gamma$ ), form a vertex $v_{i}$; arbitrarily choose an ordering, $\left(a_{i}, b_{i}\right)$, to be the default ordering of the pair.

2. For each triple $t_{i}=\left\{x_{i}, y_{i}, z_{i}\right\}$ in the full orbit presentation, form a vertex $w_{i}$; arbitrarily choose a cyclic ordering $\left\{\left(x_{i}, y_{i}\right),\left(y_{i}, z_{i}\right),\left(z_{i}, x_{i}\right)\right\}$ to be the standard triple for $t_{i}$.

The standard triple is not a transitive triple, nor is its converse. Thus we require in essence that the pairs not be directed so as to give a standard triple or its converse. To guarantee this, we connect the $\left\{v_{i}\right\}$ and $\left\{w_{j}\right\}$ as follows:

3. For each triple $t_{i}=\left\{x_{i}, y_{i}, z_{i}\right\}$, let $p_{i 1}, p_{i 2}, p_{i 3}$ be the orbit representatives for the orbits containing the pairs $\left\{x_{i}, y_{i}\right\},\left\{y_{i}, z_{i}\right\}$ and $\left\{x_{i}, z_{i}\right\}$ respectively. (These pair orbits need not be distinct.) Now for $j \in\{1,2,3\}$, if the standard triple for $t_{i}$ contains a pair in the orbit of the default pair of $p_{i j}$, place an edge between the vertex $v_{i j}$ for pair $p_{i j}$ and the vertex $w_{i}$. Otherwise add a path of length two between $v_{i j}$ and $w_{i}$, by adding a new vertex $m_{i j}$ and edges $\left\{\left\{v_{i j}, m_{i j}\right\},\left\{m_{i j}, w_{i}\right\}\right\}$.

In $G$, every vertex $w_{i}$ has degree 3 by construction; a vertex $v_{i}$ has degree $2 \lambda$ if, for the corresponding pair $p_{i}=\left\{a_{i}, b_{i}\right\}$, the orbit of $\left\{a_{i}, b_{i}\right\}$ has the same size as the orbit of $\left(a_{i}, b_{i}\right)$. In this case, $\{x, y\}$ has a full orbit. Otherwise $v_{i}$ has degree $\lambda$, and the 
pair has a half orbit; this reflects the fact that the orbit of $p_{i}$ is accounted for twice by each occurrence of a pair in a full orbit of triples in the full orbit presentation. Our assumption that $\Gamma$ has a planar action ensures that a half orbit of pairs only arises by fixing one point of a triple and interchanging the other two.

We next colour the edges of $G$ with two colours, so that

(i) for each vertex $v_{i}$, no more than $\lambda$ of the edges incident at $v_{i}$ receive the same colour;

(ii) for each vertex $w_{i}$, the three edges incident at $w_{i}$ do not all receive the same colour; and

(iii) for each intermediate vertex $m_{i j}$ on a path of length two from a vertex in $\left\{v_{i}\right\}$ to one in $\left\{w_{j}\right\}$, the two edges incident at $m_{i j}$ receive different colours.

To produce such a colouring, we proceed as follows. We use two colours, say black and white. Initially all edges of $G$ are uncoloured. As long as there is an uncoloured edge incident at a vertex $w_{i}$, proceed as follows. Form a maximal trail ("path" with repeated vertices allowed) of uncoloured edges starting at $w_{i}$. This trail can terminate either at $w_{j}, j \neq i$, or at a vertex $v_{k}$ of degree $\lambda$. In either event, alternate colouring black and white along the trail, starting with a colour that has not yet appeared at $w_{i}$ if possible. Repeating this results in a colouring of all edges of $G$. For the resulting colouring, conditions (i) and (iii) are immediate. We must check condition (ii). Consider a vertex $w_{i}$. If $w_{i}$ appears as an interior vertex on any of the maximal trails chosen to colour, the two consecutive edges on this trail incident at $w_{i}$ receive different colours. If instead $w_{i}$ appears as the first vertex of two or more of the chosen trails, in the second of these the edge at $w_{i}$ receives a different colour than does the edge at $w_{i}$ in the first. Finally, $w_{i}$ cannot be the last vertex in two or more trails, since trails are chosen to be maximal.

Given a 2-edge colouring of $G$ satisfying (i), (ii) and (iii), we now describe how to direct the system. For each triple $t_{i}=\{x, y, z\}$ in the full orbit presentation, let $\{(x, y),(y, z),(z, x)\}$ be the standard triple for $t_{i}$. Let the three edges $e_{1}, e_{2}, e_{3}$ incident at $w_{i}$ correspond to the edge for the pairs $\{x, y\},\{y, z\}$, and $\{x, z\}$ in $t_{i}$, respectively. Now form a transitive triple $t^{\prime}$ containing

$$
\begin{aligned}
& (x, y) \text { if } e_{1} \text { is white, }(y, x) \text { if } e_{1} \text { is black; } \\
& (y, z) \text { if } e_{2} \text { is white, }(z, y) \text { if } e_{2} \text { is black; and } \\
& (z, x) \text { if } e_{3} \text { is white, }(x, z) \text { if } e_{3} \text { is black. }
\end{aligned}
$$

We claim that $t^{\prime}$ is a transitive triple, since of the eight possible ways to order the pairs of $t_{i}$, the only nontransitive triples occur when all of $e_{1}, e_{2}, e_{3}$ receive the same colour.

Replacing every triple $t_{i}$ in this way certainly yields a collection of orbits of transitive triples under $\Gamma$. We must also verify that every ordered pair appears in precisely 
$\lambda$ triples. Now consider an orbit of pairs represented by the vertex $v_{i}$. If the orbit is full, $v_{i}$ has degree $2 \lambda$. Moreover, $\lambda$ edges incident at $v_{i}$ are white and $\lambda$ are black. By construction, every white edge at $v_{i}$ signifies that the corresponding triple has used the default ordering of the pair, while each black edge signifies one use of the reverse of the default ordering.

If $v_{i}$ corresponds to a half orbit of pairs, each occurrence of the default ordered pair in an orbit of transitive triples is matched by an occurrence of the reverse of the default in the same orbit of triples. Thus every orbit of ordered pairs is accounted for precisely $\lambda$ times as required.

Corollary 3.2. A $T S(v, 2)$ with group $\Gamma$ underlies a $D T S(v, 1)$ with group $\Gamma$ only if $\Gamma$ has a planar action on the $T S$, and the $T S$ has a full orbit presentation under $\Gamma$.

\section{Consequences}

Theorem A, that every $T S(v, 2 \lambda)$ is directable, is an easy consequence of Theorem 3.1, taking $\Gamma$ to be the identity group. Moreover, in the proof of Theorem 3.1, the 2-edge-colouring of the graph $G$ can easily be produced in time that is linear in the size of $G$, and hence Theorem 3.1 leads to a linear time algorithm for directing triple systems that appears quite different from the linear time algorithm of [12].

Taking $\Gamma$ to be the cyclic group $Z_{v}$ for a $T S(v, 2 \lambda)$ in Theorem 3.1 gives:

Proposition 4.1. A cyclic $T S(v, 2 \lambda)$ with all orbits of sizes $v$ and $v / 3$ can be directed to form a cyclic $D T S(v, \lambda)$ if and only if the number of triple orbits of size $v / 3$ is a multiple of 3 .

Harms [11] proved Proposition 4.1 when $\lambda=1$, giving an $O\left(v^{3}\right)$ algorithm for directing in this case. The proof of Theorem 3.1 gives an $O(\lambda v)$ time algorithm for all $\lambda$. Moreover, using the existence proofs for cyclic $T S(v, 2 \lambda)$ in [7], we obtain the result of Cho, Han and Kang [3]:

Proposition 4.2. A cyclic DTS $(v, \lambda)$ exists if and only if

(i) $\lambda \equiv 1,5(\bmod 6)$ and $v \equiv 1,4,7(\bmod 12)$;

(ii) $\lambda \equiv 2,4(\bmod 6)$ and $v \equiv 1(\bmod 3)$;

(iii) $\lambda \equiv 3(\bmod 6)$ and $v \equiv 0,1,3(\bmod 4)$; or

(iv) $\lambda \equiv 0(\bmod 6)$ and $v \geqslant 3$.

A similar proposition for 1-rotational systems can be established. Cho [1] proves that there is a 1-rotational $T S(v, \lambda)$ with even $\lambda$ whenever $v \equiv 0,1(\bmod 3)$ and $\lambda \equiv 2,4(\bmod 6)$, or $v \equiv 2(\bmod 3)$ and $\lambda \equiv 0(\bmod 2)$. When $v \equiv 1(\bmod 3)$ and $\lambda \equiv 2,4(\bmod 6)$, there must be a short orbit of triples that cannot be repeated a 
number of times that is a multiple of 3 . Hence in these cases, the $T S$ cannot underly a DTS having the 1-rotational automorphism. Using Cho's remaining constructions for 1-rotational $T S(v, \lambda)$ 's, together with Theorem 3.1, we obtain a new result:

Lemma 4.3. A 1-rotational DTS $(v, \lambda)$ exists if and only if

(i) $\lambda \equiv 0(\bmod 3)$ and $v \equiv 1,2(\bmod 3)$; or

(ii) $v \equiv 0(\bmod 3)$.

Cho, Chae and Hwang [2] give a similar result for $k$-rotational $D T S(v, 1)$ 's, that can also be obtained by applying Theorem 3.1 .

Finally, we remark that there are numerous constructions for $T S(v, 2 \lambda)$ 's with larger groups; Theorem 3.1 enables us to carry over the large automorphism groups in whole or in part to directed triple systems.

\section{REFERENCES}

[1] C.J. Cho, 'Rotational triple systems', Ars Combin. 13 (1982), 203-209.

[2] C.J. Cho, Y. Chae and S.G. Hwang, 'Rotational directed triple systems', J. Korean Math. Soc. 24 (1987), 133-142.

[3] C.J. Cho, Y.-H. Han and S.-H. Kang, 'Cyclic directed triple systems', J. Korean Math. Soc. 23 (1986), 117-125.

[4] C.J. Colbourn and M.J. Colbourn, 'Every twofold triple system can be directed', J. Combin. Theory A34 (1983), 375-378.

[5] C.J. Colbourn and J.J. Harms, 'Directing triple systems', Ars Combin. 15 (1983), 261-266.

[6] M.J. Colbourn and C.J. Colbourn, 'Some small directed triple systems', Congr. Numer. 30 (1981), 247-255.

[7] M.J. Colbourn and C.J. Colbourn, 'Cyclic block designs with block size 3', European J. Combin. 2 (1981), 21-26.

[8] M.J. Colbourn and C.J. Colbourn, 'The analysis of directed triple systems by refinement', Ann. Discrete Math. 15 (1982), 97-103.

[9] M.J. Colbourn and R.A. Mathon, 'On cyclic Steiner 2-designs', Ann. Discrete Math. 7 (1980), 215-253.

[10] J. Doyen and A. Rosa, 'An updated bibliography on Steiner systems', Ann. Discrete Math. 7 (1980), 317-349. Unpublished update February, 1989.

[11] J.J. Harms, 'Directing cyclic triple systems', Ann. Discrete Math. 26 (1985), 221-225.

[12] J.J. Harms and C.J. Colbourn, 'An optimal algorithm for directing triple systems using eulerian circuits', Ann. Discrete Math. 27 (1985), 433-438.

[13] S.H.Y. Hung and N.S. Mendelsohn, 'Directed triple systems', J. Combin. Theory A14 (1973), 310-318.

[14] J. Seberry and D. Skillicorn, 'All directed BIBDs with $k=3$ exist', J. Combin. Theory A29 (1980), 244-248. 
[15] D.R. Stinson and W.D. Wallis, 'Snappy constructions for triple systems', Austral. Math. Soc. Gaz. 10 (1983), 84-88.

Department of Combinatorics and Optimization

University of Waterloo

Waterloo, Ontario

Canada N2L 3G1 